Article

\title{
Resonant Tunneling Diode (RTD) Terahertz Active Transmission Line Oscillator with Graphene-Plasma Wave and Two Graphene Antennas
}

\author{
Fan Zhao ${ }^{1}\left(\mathbb{D}\right.$, Changju Zhu ${ }^{1}$, Weilian Guo ${ }^{2}$, Jia Cong ${ }^{1}$, Clarence Augustine T. H. Tee ${ }^{3}{ }^{\circledR}$, \\ Le Song ${ }^{4}\left(\mathbb{D}\right.$ and Yelong Zheng ${ }^{4, *}$ \\ 1 Department of Microelectronics and Solid-state Electronics, School of Electrical and Information Engineering, \\ Tianjin University, Tianjin 300072, China; fanhere@tju.edu.cn (F.Z.); chedanzhuzhu@126.com (C.Z.); \\ congj2012@163.com (J.C.) \\ 2 Department of Microelectronics and Solid-state Electronics, School of Microelectronics, Tianjin University, \\ Tianjin 300072, China; wlg886688@163.com \\ 3 Department of Electrical Engineering, Faculty of Engineering, University of Malaya, \\ Kuala Lumpur 50603, Malaysia; catht@um.edu.my \\ 4 State Key Laboratory of Precision Measuring Technology and Instruments, Tianjin University, \\ Tianjin 300072, China; songle@tju.edu.cn \\ * Correspondence: zhengyelongby@tju.edu.cn; Tel.: +86-182-2233-3873
}

Received: 14 September 2019; Accepted: 11 October 2019; Published: 14 October 2019

check for updates

\begin{abstract}
This study describes the design of a resonant tunneling diode (RTD) oscillator (RTD oscillator) with a RTD-gated-graphene-2DEF (two dimensional electron fluid) and demonstrates the functioning of this RTD oscillator through a transmission line simulation model. Impedance of the RTD oscillator changes periodically when physical dimension of the device is of considerable fraction of the electrical wavelength. As long as impedance matching is achieved, the oscillation frequency is not limited by the size of the device. An RTD oscillator with a graphene film and negative differential resistance (NDR) will produce power amplification. The positive electrode of the DC power supply is modified and designed as an antenna. So, the reflected power can also be radiated to increase RTD oscillator output power. The output analysis shows that through the optimization of the antenna structure, it is possible to increase the RTD oscillator output to $22 \mathrm{~mW}$ at $1.9 \mathrm{THz}$ and $20 \mathrm{~mW}$ at 6.1 THz respectively. Furthermore, the RTD oscillator has the potential to oscillate at $50 \mathrm{THz}$ with a matching antenna.
\end{abstract}

Keywords: resonant tunneling diode (RTD), terahertz; two-dimensional electron fluid (2DEF), RTD-gated-graphene-2DEF; graphene-plasma; resonant tunneling diode oscillator (RTD oscillator), transmission line model

\section{Introduction}

Terahertz (THz) electromagnetic radiation is generally composed of electromagnetic waves with frequencies of $100 \mathrm{GHz}-10 \mathrm{THz}$ [1]. The wavelengths of THz waves fall between those of light waves and millimeter waves. They are characterized by strong penetration, high security, and good orientation, and they can be used extensively in medical and exploration applications [2,3]. However, a coherent source with high-power work at room temperature is indispensable for these applications. Terahertz wave sources are classified into optical wave sources and electrical wave sources. Optical THz products have been available commercially [4-7], but they are expensive and bulky. Resonant tunneling diodes (RTDs) [8,9] are good candidates for electronic $\mathrm{THz}$ wave sources from the viewpoint of fabricating resonant tunneling diode oscillator (RTD oscillators). Resonant tunneling diode oscillators 
are coherent, solid-state, and compact high-power sources with high-power work at room temperature. H. Kanaya et al. [2] reported a $1.42 \mathrm{THz}$ oscillation via a reduction in the RTD intrinsic delay. The same team reported a $1.55 \mathrm{THz}$ oscillation through optimization of the antenna length [10]. A $1.98 \mathrm{THz}$ RTD oscillator was fabricated with the optimum electrode thickness of $2 \mu \mathrm{m}$ [3]. This is the highest RTD oscillator frequency reported so far. In reference [4], the authors used a conventional lumped-element equivalent circuit to analyze a RTD oscillator. A potential oscillation of $3.0 \mathrm{THz}$ was shown with the RTD and also at $2.8 \mathrm{THz}$ for the integrated device with a slot antenna while the device structure was optimized. However, the theoretical analysis showed that oscillator frequency and RTD size were correlated negatively [4]. By contrary, the device output power and RTD size were correlated positively. Asada, et al. [11] showed in their earlier work, the oscillation frequency increased to $345 \mathrm{GHz}$ with a slot width of $2 \mathrm{~mm}$ from a $330 \mathrm{GHz}$ with a slot width of $4 \mathrm{~mm}$. For [3], the oscillation frequency of the RTD Oscillator is already close to $2 \mathrm{THz}$. At such high frequencies, transmission line theory is more suitable for analyzing RTD Oscillator than the conventional lumped-element equivalent circuit model. Transmission line theory bridges the gap between field analysis and basic circuit theory and therefore is of significant importance in the analysis of microwave even terahertz circuits and devices. The phenomenon of wave propagation on transmission lines can be approached from an extension of circuit theory or from a specialization of Maxwell's equations [12]. Moreover, the fabrication of high frequency RTD oscillator remains challenging.

In [13], Burke et al. developed the high-frequency transmission line model to describe a metal-gate/insulator/two-dimensional electron fluid (2DEF) structure. Then, the model was used in $\mathrm{THz}$ power amplification via plasma wave excitation [14,15], high-electron-mobility transistor (HEMT) with RTD gate [16], and photo-controlled THz amplified modulator [17]. In the previous study, a graphene layer was employed for plasma wave excitation [18-21]. Optical graphene plasmon resonances were realized spectroscopically with a two-dimensional graphene sheet, and propagating plasmons in real space were resolved directly in experiments.

The study here describes the design of a resonant tunneling diode (RTD) oscillator (RTD oscillator) with a RTD-gated-graphene-two-dimensional electron fluid (2DEF) and demonstrates the functioning of this RTD oscillator through a transmission line model simulation. Firstly, the distributed transmission line model analysis in this study shows that the size of the device is not an indefinite factor affecting the oscillation frequency. The impedance of the device changes periodically when physical dimension of the device is of considerable fraction of the electrical wavelength. As long as impedance matching is achieved, the oscillation frequency is not limited by the size of the device. Therefore, without reducing the frequency, the output power can be greatly increased with the increase of the device size. Secondly, output power increase is not only due to the increase in device size, but also due to a graphene layer which produces RTD-gated-graphene-2DEF and the negative differential resistance (NDR). According to references $[14,16,17]$, two-dimensional electron fluid (2DEF) is required to realize power amplification, which is produced by RTD-gated HEMT with very complicated layered structure. Because the films thickness of the RTD-gated HEMT are very small, only a few nanometers, so the molecular beam epitaxy [19,22,23] or metal-organic chemical vapor deposition [24] is required [25]. They are complex and expensive processes. Due to the graphene plasma phenomenon, two-dimensional electron fluid (2DEF) is generated by a graphene film instead of RTD-gated HEMT, reducing the difficulty of implementing the RTD oscillator. Thirdly, transmission lines model in this study shows that power reflection cannot be completely eliminated, both in theory and in practical applications. This is especially when impedance matching is not satisfied, power reflection and loss would be large. A DC bias is needed to make the device work normally. RTD requires two electrodes and oscillator requires an antenna. Refer to $[2,3,10]$ and [26], it is common to apply a DC bias voltage to the antenna. In this study, the positive electrode of the DC power supply is modified and designed as an antenna. So, the reflected power can also be radiated to increase RTD oscillator output power. In this study, the Authors demonstrated RTD oscillator could output power of $22 \mathrm{~mW}$ at $1.9 \mathrm{THz}$ and $20 \mathrm{~mW}$ at $6.1 \mathrm{THz}$ respectively. 


\section{Modeling}

A RTD is a semiconductor that exhibits controllable negative difference resistance (NDR) regions. The N-shaped I-V characteristics $[27,28]$ of RTDs provide electrical gain for THz oscillator at room temperature [14]. A RTD is realized via the creation of a double-barrier quantum well (DBQW) structure [26] comprising a narrow-bandgap material sandwiched between two thin slabs of a wide-bandgap material. However, the results of a theoretical analysis show that oscillator frequency and RTD size are negatively correlated [4]. In this study, a graphene layer is fabricated before the sandwich structure, and the graphene layer forms a RTD-gated-graphene-2DEF. The developed RTD oscillator realizes power amplification via a graphene-plasma wave layer [17]. As shown in Figure 1a,b, a RTD oscillator comprises a RTD, RTD-gated-graphene-2DEF and two graphene antennas. As shown in Figure $1 b$, the length of a transmission line is equal to the channel length $L$, which includes a RTD and a RTD-gated-graphene-2DEF.As shown in Figure 1b, a distributed circuit model is established to represent the contributions of NDR and 2DEF to a RTD-gated-graphene-2DEF oscillator. The RTD oscillator is biased by the application of dc voltage to the left antenna [29].

Electrical size is key difference between transmission line model and lumped-element equivalent circuit model [12]. It assumes that circuit analysis works when physical dimension of the device is much smaller than the electrical wavelength, while transmission lines model works when physical dimension of the device is a considerable fraction of the electrical wavelength. Electrical wavelength is wavelength of alternating current signals, which is defined as $\lambda=c / f$, where $c$ is speed of light, $f$ is frequency of alternating current signal. Electrical size is defined as $k=W / \lambda$, where $W=10 \mu \mathrm{m}$ is the RTD width in this study [12]. The oscillator frequency ranges from $1 \mathrm{THz}$ to $10 \mathrm{THz}, 1 / 30 \leq k \leq 1 / 3$. A transmission line model is a network with distributed parameters, where currents and voltages vary in magnitude and phase over its length. Current and voltage of conventional lumped-element equivalent circuit do not vary appreciably over the size of the device.

As shown in Figure 1b,c, transmission line model is represented as a two-wire line since transmission lines always have at least two conductors. The following parameters are used to describe the distributed circuit model of a transmission line: $r$ is series resistance per unit length, for both conductors, in $\Omega / \mathrm{cm}, c$ is shunt capacitance per unit length, in F/cm, $g$ is shunt conductance per unit length, in $\mathrm{S} / \mathrm{cm}, l$ is series inductance per unit length, for both conductors, in $\mathrm{H} / \mathrm{cm}$.

These parameters are defined as $[12,14,16,17]$

$$
\begin{gathered}
r=\frac{1}{e \mu n_{s} W} \\
c=c_{0} W \\
g=g_{0} W=W \frac{d I}{d V} \\
l=\frac{m^{*}}{e^{2} \mu n_{s} W}
\end{gathered}
$$

where $e$ is the electronic charge, in $1.6^{*} 10^{-19} \mathrm{C}$; $\mu$ is the electron mobility in the transmission line, in $\mathrm{cm}^{2} \mathrm{~V}^{-1} \mathrm{~s}^{-1} ; W$ the RTD width, in $\mathrm{cm} ; m^{*}$ is the electron effective mass, in $\mathrm{kg} ; n_{s}$ is the 2DEF sheet charge density, in $\mathrm{cm}^{-2} ; g_{0}$ is the gate conductance per unit area, in $\mathrm{S} / \mathrm{cm}^{2}$; and $c_{0}$ is the gate capacitance per unit area, in $\mathrm{F} / \mathrm{cm}^{-2}$. The complex propagation constant $\gamma$ of the transmission line model is defined as [12]

$$
\gamma=\sqrt{(r+j \omega l)(g+j \omega c)}=\alpha+j \beta
$$

where $\omega$ is the angular frequency. The characteristic impedance $Z_{0}$ is defined as [26]

$$
Z_{0}=\sqrt{\frac{r+j \omega l}{g+j \omega c}}
$$




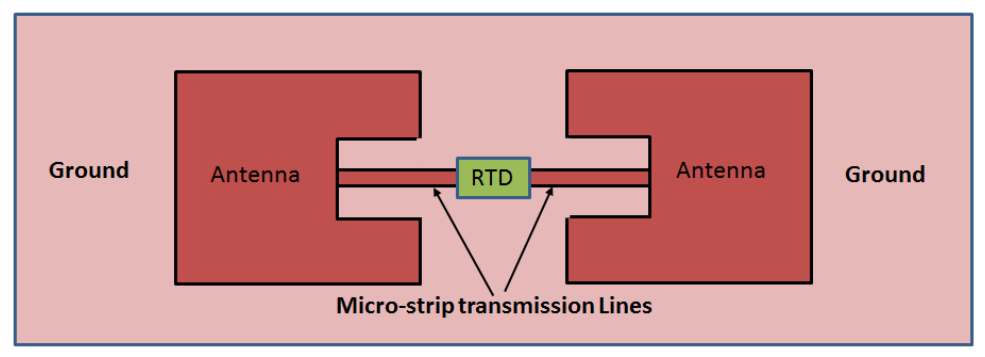

(a)

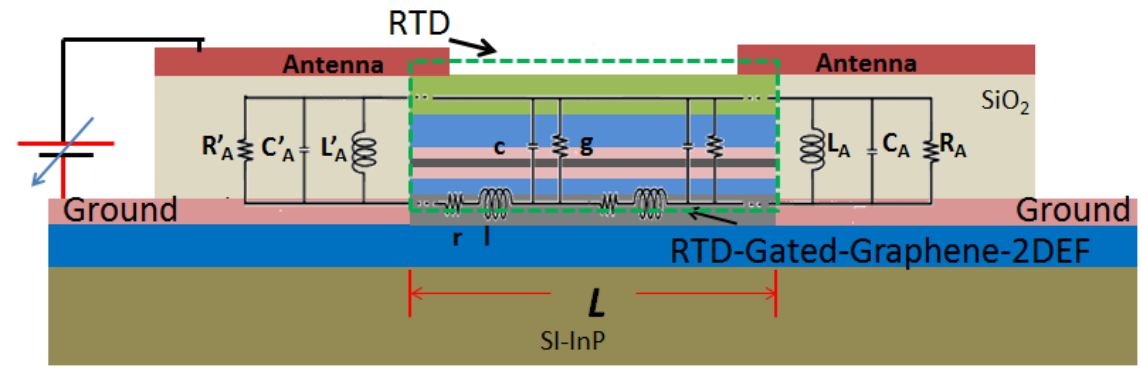

(b)

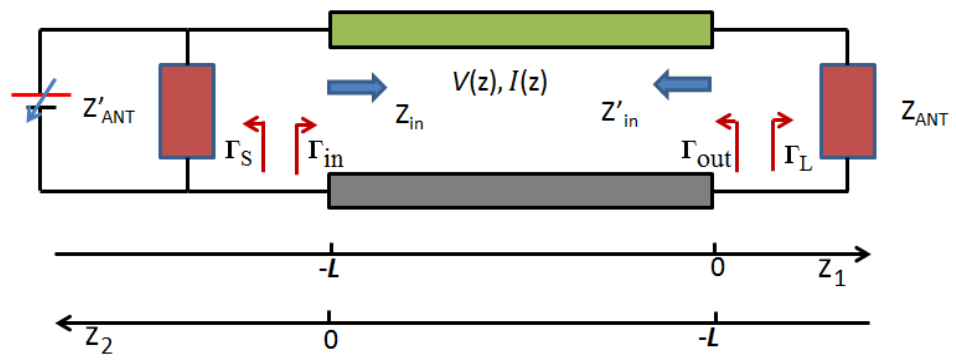

(c)

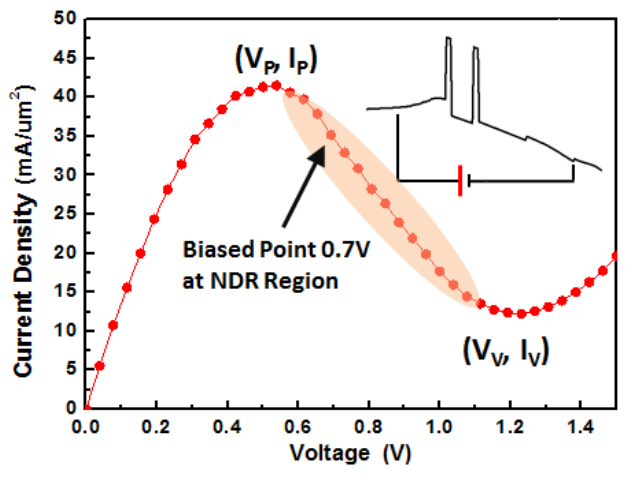

(d)

Figure 1. (a) Top view of resonant tunneling diode (RTD) terahertz oscillator. The developed oscillator comprises a RTD, RTD-gated-graphene-2DEF, and two graphene antennas. (b) Cross-section of RTD terahertz oscillator. Distributed transmission line model of RTD-gated-graphene-2DEF (two dimensional electron fluid) RTD oscillator. (c) Equivalent active transmission line oscillator model. (d) I-V characteristic of the RTD. 
Figure 1 Equivalent active transmission line oscillator model shows a length $\mathrm{L}$ of a lossy transmission line terminated in a right antenna impedance $Z_{A N T}$. Thus, $\gamma=\alpha+j \beta$ is complex, but $Z_{0}$ is approximately real when we assume the loss is small. The expressions for current and voltage wave on the lossy line are.

$$
\begin{aligned}
& I(z)=\frac{V_{0}^{+}}{Z_{0}}\left[e^{-\gamma z}-\Gamma e^{\gamma z}\right] \\
& V(z)=V_{0}^{+}\left[e^{-\gamma z}-\Gamma e^{\gamma z}\right]
\end{aligned}
$$

As shown in Equations (7) and (8), where $\Gamma$ is the reflection coefficient of the load. At $z=0, V_{0}{ }^{+}$is the incident voltage amplitude. At a distance $L$ from the load, the reflection coefficient is

$$
\Gamma(L)=\frac{V_{0}^{-} e^{-j \beta L}}{V_{0}^{+} e^{j \beta L}} e^{-2 \alpha L}=\Gamma(0) e^{-2 \alpha L} e^{-2 j \beta L}=e^{-2 \gamma L}
$$

$\Gamma(0)$ is the reflection coefficient at $\mathrm{z}=0$, as

$$
\Gamma(0)=\frac{V_{0}^{-}}{V_{0}^{+}}=\frac{Z_{A N T}-Z_{0}}{Z_{A N T}+Z_{0}}
$$

According to axis $\mathrm{z}_{1}$, the impedance $Z_{\text {in }}$ toward the right of the transmission line model of RTD oscillator is as

$$
Z_{\text {in }}=\frac{V(-L)}{I(-L)}=Z_{0} \frac{Z_{A N T}+\mathrm{j} Z_{0} \tanh \gamma L}{Z_{0}+j Z_{A N T} \tanh \gamma L}
$$

The impedance of the left antenna is $Z_{A N T}^{\prime}$. According to axis $z_{2}$, the impedance $Z_{\text {in }}^{\prime}$ toward the left is

$$
Z^{\prime}{ }_{\text {in }}=\frac{V(-L)}{I(-L)}=Z_{0} \frac{Z^{\prime}{ }_{A N T}+\mathrm{j} Z_{0} \tanh \gamma L}{Z_{0}+j Z^{\prime}{ }_{A N T} \tanh \gamma L}
$$

In this study, two antennas of the same size were designed. The impedances $Z_{A N T}$ and $Z_{A N T}^{\prime}$ are equal, and the impedances $Z_{\text {in }}$ and $Z_{\text {in }}^{\prime}$ are equal.

$$
\begin{aligned}
Z_{A N T} & =Z_{A N T}^{\prime} \\
Z_{\text {in }} & =Z_{\text {in }}^{\prime}
\end{aligned}
$$

To ensure that the oscillator works, Equation (15) must be satisfied as following

$$
\operatorname{Re}\left(Z_{i n}\right)+\operatorname{Re}\left(Z_{A N T}^{\prime}\right)=0
$$

$\operatorname{Re}\left(Z_{\text {in }}\right)$ is the real part of $Z_{i n}$, and $\operatorname{Re}\left(Z_{A N T}^{\prime}\right)$ is the real part of $Z_{A N T}^{\prime}$. I-V characteristic of RTD which was simulated by WINGREEN is shown in Figure $1 \mathrm{~d}$. This is a N-type NDR, and $\mathrm{g}$ is called negative difference conductor (NDC) which is defined as [16]

$$
g=\frac{\Delta I}{\Delta V}=\frac{I_{P}-I_{V}}{V_{P}-V_{V}}
$$

where $I_{P}$ is peak current density, $I_{V}$ is valley current density, $V_{P}$ is peak voltage, $V_{V}$ is valley voltage. As shown in Figure $1 \mathrm{~d}, \Delta I=29 \mathrm{~mA} / \mu \mathrm{m}^{2}$ and $\Delta V=0.7 \mathrm{~V}$. The RTD oscillator is biased at $0.7 \mathrm{~V}$ at NDR region. According to reference $[14,16,17]$, Equation (3) can be assumed as

$$
g=-r c / l
$$

The load achieves the maximum power transmission when the loaded impedance $Z_{A N T}$ and power source internal resistance $Z^{\prime}{ }_{\text {in }}$ for conjugate match in sine stable state electric circuit. To achieve the maximum power transmission, stable oscillation should satisfy the following condition. 


$$
Z_{\text {in }}^{\prime}=Z_{A N T}^{*}
$$

According to Equations (14)-(18), to achieve the maximum power transmission, $\operatorname{Re}\left(Z_{\text {in }}\right)$ and $\operatorname{Re}\left(Z^{\prime}{ }_{A N T}\right)$ must be equal as well. So, $\operatorname{Re}\left(Z_{\text {in }}\right)$ must equals to "zero", as shown in Figures 2 and 3.

$$
\operatorname{Re}\left(Z_{\text {in }}\right)=\operatorname{Re}\left(Z_{A N T}\right)=0
$$

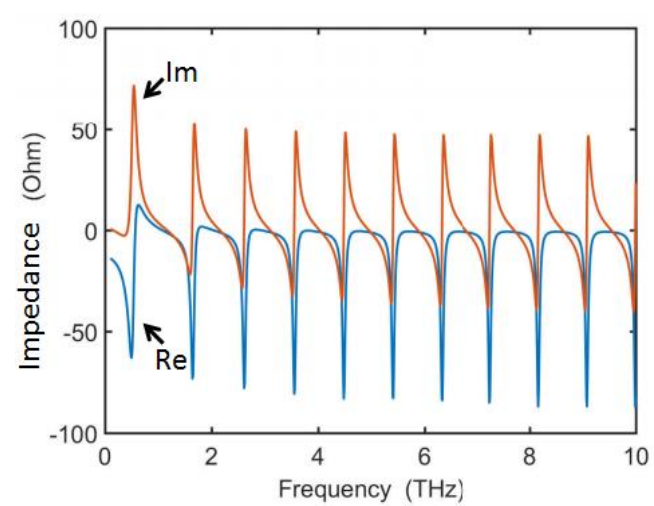

(a)

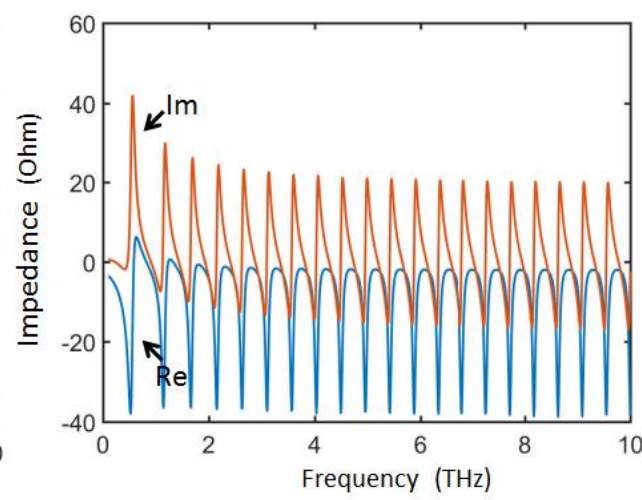

(b)

Figure 2. Relationship between impedance and frequency of transmission line: (a) $Z_{\text {in }}$ or $Z_{\text {in }}^{\prime}(L=1 \mu \mathrm{m})$ and (b) $Z_{\text {in }}$ or $Z_{\text {in }}^{\prime}(L=2 \mu \mathrm{m})$.

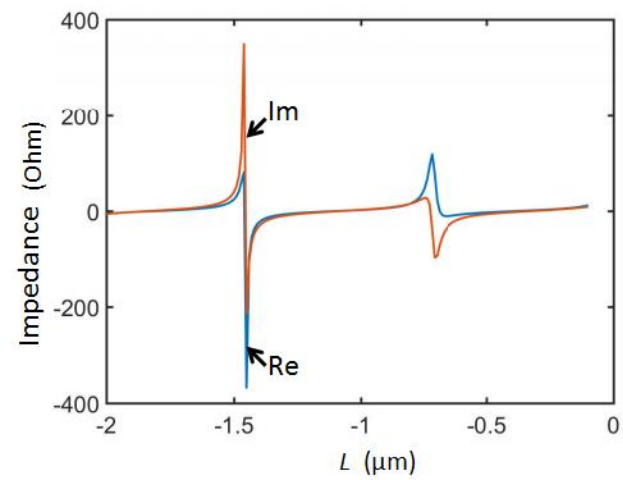

(a)

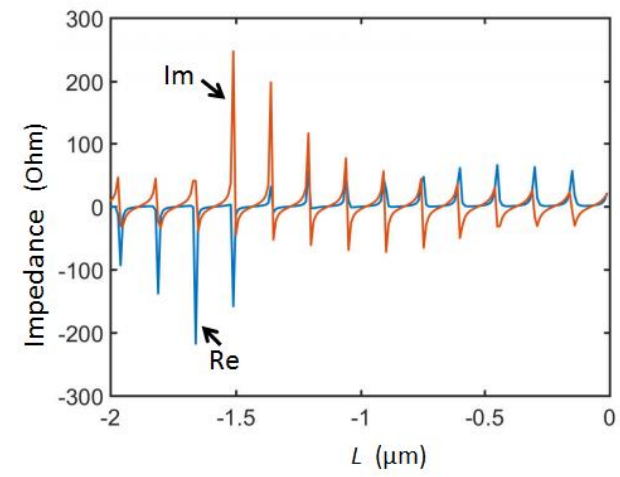

(c)

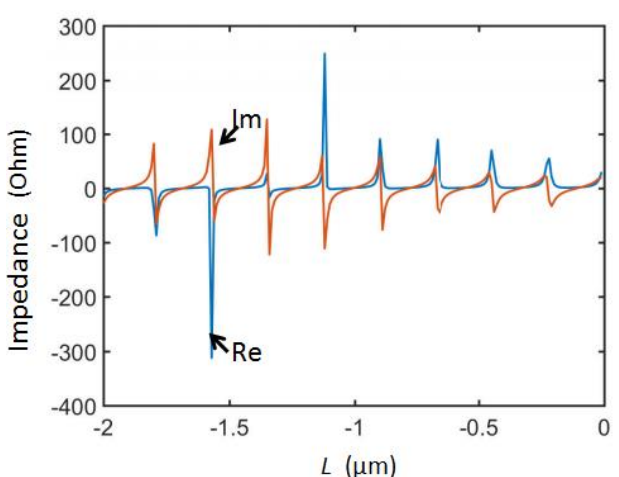

(b)

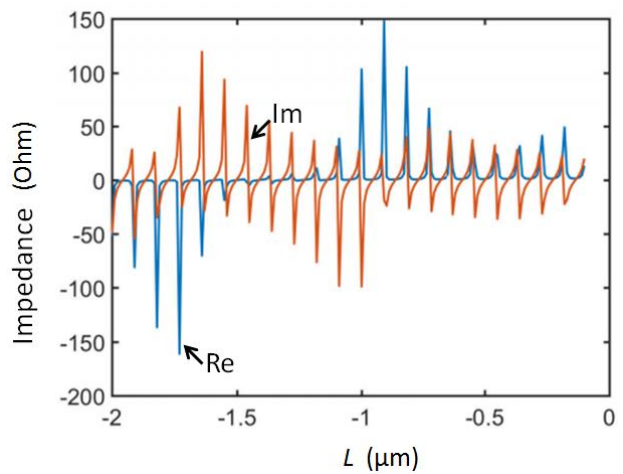

(d)

Figure 3. Relationship between impedance $Z_{\text {in }}$ or $Z_{\text {in }}^{\prime}$ and length of transmission line. (a) Frequency $=$ $0.1 \mathrm{THz},(\mathbf{b})$ frequency $=1 \mathrm{THz},(\mathbf{c})$ frequency $=6 \mathrm{THz}$, and $(\mathbf{d})$ frequency $=10 \mathrm{THz}$. 
$\mathrm{G}_{\mathrm{T}}$ denotes transducer power gain. Because of the plasma wave in the RTD-gated-graphene-2DEF and the negative differential conductance (NDC) of the RTD, according to the transmission line theory, the oscillator works and amplifies $\mathrm{THz}$ waves in the channel [13], as

$$
G_{T}=\frac{P_{L}}{P_{a v s}}=\frac{\left|S_{22}\right|^{2}\left(1-\left|\Gamma_{S}\right|^{2}\right)\left(1-\left|\Gamma_{L}\right|^{2}\right)}{\left|1-\Gamma_{S} \Gamma_{i n}\right|^{2}\left|1-S_{22} \Gamma_{L}\right|^{2}}
$$

where $P_{L}$ is the power transmitted to the load, and $P_{a v s}$ is the power available from the source, which is the maximum power that can be supplied to the transmission line. S11 is the return loss, and S21 is the transmission gain. $\Gamma_{\mathrm{S}}$ and $\Gamma_{\mathrm{L}}$ are the source reflection coefficient and the load reflection coefficient, respectively. Assuming no reflection, $\Gamma_{\mathrm{S}}=\Gamma_{\mathrm{L}}=0$, and Equation (20) can be simplified as

$$
G_{T}=\left|S_{21}\right|^{2}
$$

In Equations (20) and (21), [14]

$$
\begin{gathered}
S_{11}=S_{22}=\frac{\zeta \sinh (\gamma L)-\zeta^{-1} \sinh (\gamma L)}{2 \cosh (\gamma L)+\sinh (\gamma L)\left(\zeta+\zeta^{-1}\right)} \\
S_{12}=S_{21}=\frac{2}{2 \cosh (\gamma L)+\sinh (\gamma L)\left(\zeta+\zeta^{-1}\right)} \\
\zeta=\frac{Z_{A N T}}{Z_{0}}
\end{gathered}
$$

where the complex quantity $\zeta$ is defined as the ratio of the plasma-wave-transmission-line impedance over the source impedance. The angular frequency $\omega$ and Equation (5) can be rewritten as

$$
\begin{gathered}
\omega=\sqrt{\frac{\pi^{2}}{4 L^{2} l c}-\frac{r^{2}}{l^{2}}} \\
\gamma=\sqrt{-\frac{r^{2} c}{l}-\left(\frac{\pi^{2}}{4 L^{2} l c}-\frac{r^{2}}{l^{2}}\right) c l}=\sqrt{-\frac{\pi^{2}}{4 L^{2}}}= \pm j \frac{\pi}{2 L}
\end{gathered}
$$

Therefore, Equation (20) can be rewritten as Equation (27) [14]

$$
G_{T}=\frac{4}{\left|2 \cosh (\gamma L)+\sinh (\gamma L)\left(\zeta+\zeta^{-1}\right)\right|^{2}}=\frac{4}{\left|\left(\zeta+\zeta^{-1}\right)\right|^{2}}
$$

NDC is a fundamental factor to provide power gain under these circumstances. By inspecting (5), (6) and (27), in order to ensure at least $6 \mathrm{~dB}$ of power gain, $0<(\omega l / r)<(1 / 3)$ must be satisfied.

As shown in Figures 2 and 3, Re $\left(Z_{\text {in }}\right)$ could be zero. However, the real part of $Z_{A N T}$ is greater than zero at all times, as shown in Figure $4 \mathrm{f}$. Therefore, the right antenna cannot achieve the maximum power transmission. Consequently, RTD oscillator power is reflected toward the left. The left antenna receives the reflected power and radios it outward. As a result, the proposed RTD oscillator can achieve significantly higher power output than a traditional RTD oscillator. 


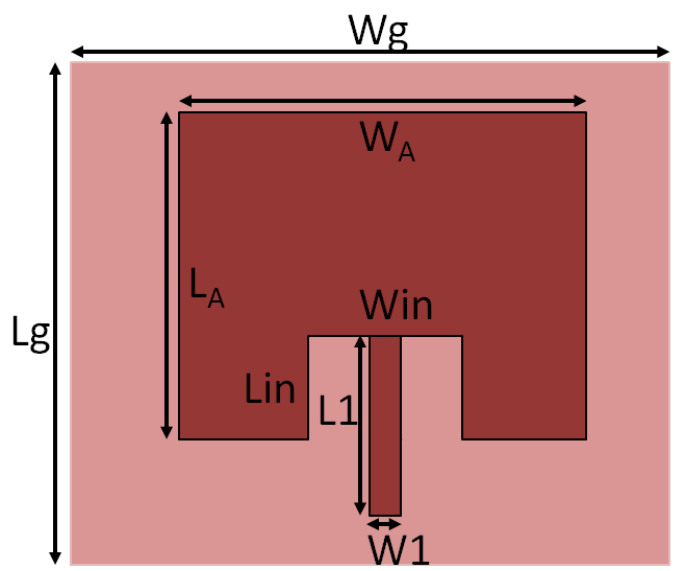

(a)

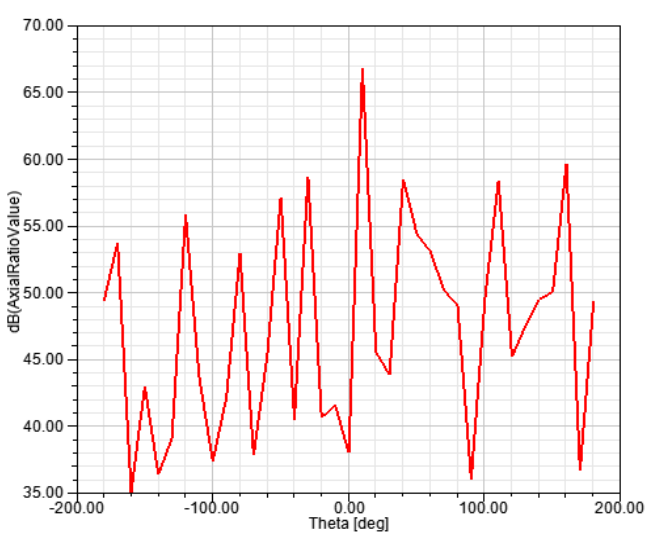

(c)

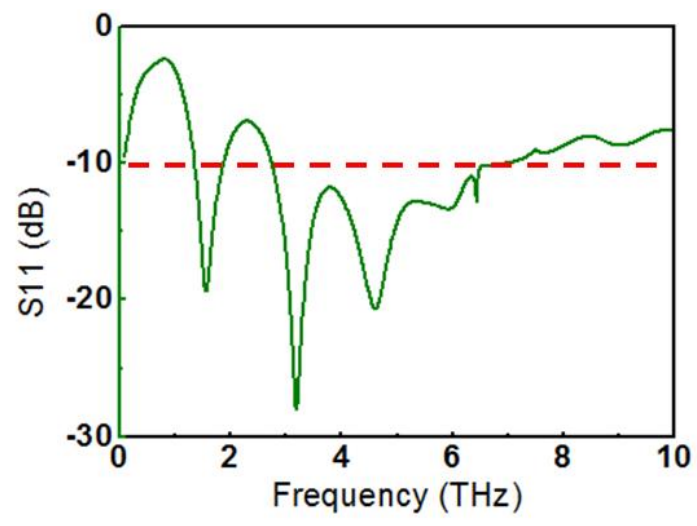

(e)
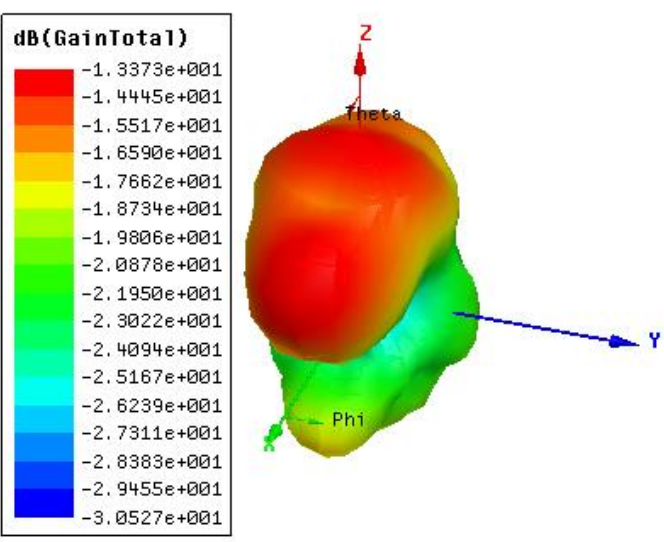

(b)
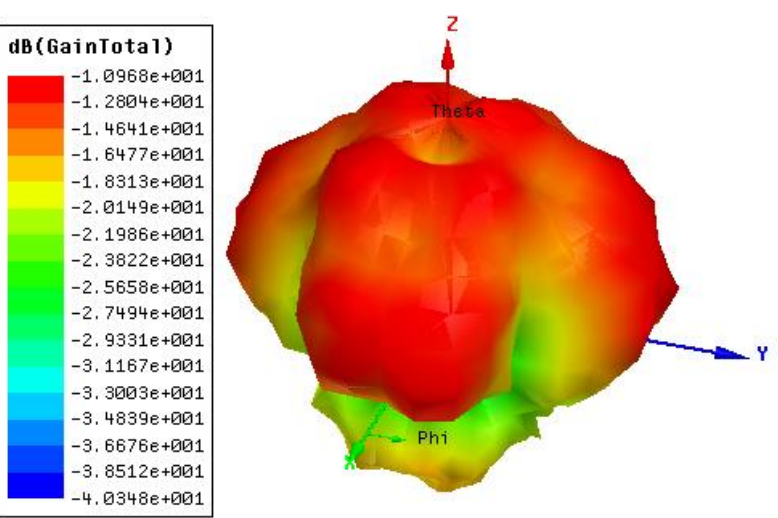

(d)

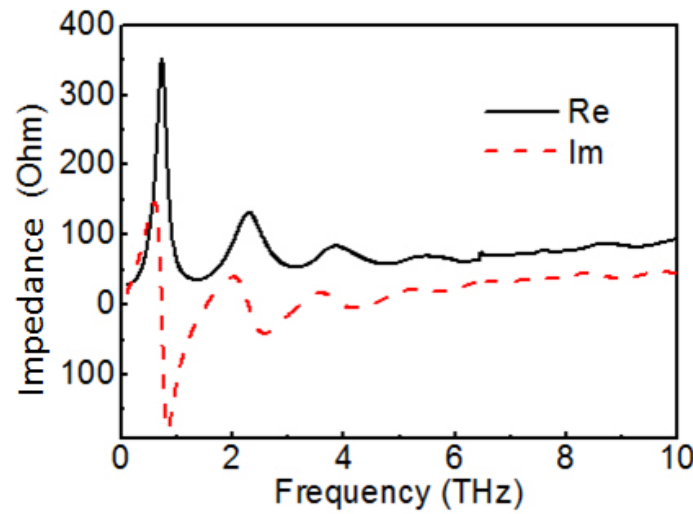

(f)

Figure 4. (a) Structure of graphene antenna $\left(\mathrm{W}_{\mathrm{A}}\right.$ is the width of the radiation patch, and $\mathrm{L}_{\mathrm{A}}$ is the length of the patch. Wg is the width of the ground plan, $\mathrm{Lg}$ the length of the ground, $\mathrm{W}_{\text {in }}$ the width of the slot, $\mathrm{L}_{\mathrm{in}}$ the length of the slot, W1 the width of the micro-strip line, and L1 the length of the micro-strip line), (b) antenna gain of single antenna, (c) axial ratio value, (d) antenna gain of two antennas, (e) S11, and (f) impedance of graphene antenna. 


\section{Simulation and Discussion}

Structural parameters in the transmission line are listed in Table 1. Figure 2 shows the relationship between the impedance and the frequency of the RTD oscillator, as derived using the transmission line model. Although the gate length of RTD is changed from $1 \mu \mathrm{m}$ to $2 \mu \mathrm{m}$, the impedance $Z_{\text {in }}$ or $Z^{\prime}{ }_{i n}$ varies periodically with the RTD oscillator frequency. Figure 3 shows that a certain impedance $Z_{\text {in }}$ or $Z^{\prime}$ in is obtained at certain RTD lengths. When the antenna impedance satisfies Equation (15), the RTD oscillator can work at multiple frequencies. To realize higher output power, the gate length must be increased. According to Equations (1)-(12) and Figure 3, increasing the gate width and the gate length of the RTD do not affect its oscillation frequency in the distributed model simulation.

Table 1. Structural parameters in the transmission line $[30,31]$.

\begin{tabular}{cc}
\hline Parameters & Value \\
\hline Electron Mobility $\mu$ & $27,000 \mathrm{~cm}^{2} \mathrm{~V}^{-1} \mathrm{~s}^{-1}$ \\
Electron Effective Mass $\mathrm{m}^{*}$ & $0.04 \mathrm{~m}_{0}$ \\
2DEG Sheet Electron Density $\mathrm{n}_{\mathrm{s}}$ & $10^{12} \mathrm{~cm}^{-2}$ \\
RTD width W & $10 \mu \mathrm{m}$ \\
\hline
\end{tabular}

As shown in Figure 4a, the two antennas in Figure 1a were designed with the same size. Figure $4 \mathrm{~b}$ shows that the antenna gain of single antenna is $-13.373 \mathrm{~dB}$. In Figure $1 \mathrm{c}$, the axial ratio value of this antenna is $38 \mathrm{~dB}$ when theta is 0 , so polarization of the antenna is linear polarization. Figure $4 \mathrm{~d}$ shows that the antenna gain of two antennas is $-10.968 \mathrm{~dB}$, which is lower than that of a regular patch antenna, because the antenna has two ultrawide bands. Antenna gain of two antennas is higher than that of single antenna. Figure $4 \mathrm{e}$ shows the S11 of the graphene antenna. When $\mathrm{S} 11<10 \mathrm{~dB}$, the antenna bands are 1.37-1.93 THz and 2.75-7.00 THz. Figure $4 \mathrm{f}$ shows the impedance of the graphene antenna.

In fact, the output power of the existing RTD oscillator terahertz source is very small, which is the main reason for its limited application. The antenna used in the prior literature is slot antenna, which is complicated in manufacturing process and is not suitable for integration with other devices and mass production. Although the graphene antenna designed in the present study has a low gain, due to the RTD-gated-graphene-2DEF of the RTD oscillator, its output power is very high. A DC bias is necessary to make the device work normally. This requires an electrode and an antenna. Refer to $[2,3,10]$ and [26], it is common to apply a DC bias voltage to the antenna. In this study, the positive electrode of the DC power supply is modified and designed as an antenna. So, the reflected power can also be radiated to increase RTD oscillator output power.

Advanced Design System (ADS, Keysight Technologies) was used to simulate the fully distributed active transmission line RTD-gated-graphene-2DEF THz oscillator in this study. As shown in Figure 5, the oscillation consists of the first and higher harmonics. The harmonic power is defined per Equations (28) to (31)

$$
\Gamma=\frac{Z_{A N T}-Z_{0}}{Z_{A N T}+Z_{0}}
$$

where $\Gamma$ denotes the voltage reflection coefficient.

The maximum output power $P$ of the transmission line can be rewritten as

$$
\begin{gathered}
|\Gamma(L)|=\Gamma e^{-2 \gamma L} \\
\alpha=\operatorname{Re}\{\gamma\} \\
P=\frac{1}{2} \operatorname{Re}\left\{V(-L) I^{*}(-L)\right\}=\frac{\left|V_{0}^{+}\right|^{2}}{2 Z_{0}}\left[1-|\Gamma(L)|^{2}\right] e^{2 \alpha L}
\end{gathered}
$$


$V_{0}{ }^{+}$is the power voltage. The generation of harmonics, including the odd harmonics, can be described by solving Equation (32)

$$
f=\frac{1+2 k}{4 \sqrt{\frac{m^{*} c_{0}}{e^{2} \mu n_{s}}} L}, k=0,1,2 \ldots \ldots,
$$

As shown in Figure 5, the power of the first harmonic is higher than that of the higher harmonics. When the device dimensions are $L=2 \mu \mathrm{m}$ and $W=10 \mu \mathrm{m}$, the power of the first harmonic is $22 \mathrm{~mW}$ at $1.9 \mathrm{THz}$ and that of the higher harmonics is $20 \mathrm{~mW}$ at $6.1 \mathrm{THz}$. When the device dimensions are $L=1 \mu \mathrm{m}$ and $W=10 \mu \mathrm{m}$, the power of the first harmonic is $11.75 \mathrm{~mW}$ at $1.9 \mathrm{THz}$ and that of the higher harmonics is $9.5 \mathrm{~mW}$ at $6.1 \mathrm{THz}$. These results show that the RTD oscillator power increases as its size increases, but its frequency is not affected. Because of the mismatch between $Z^{\prime}{ }_{\text {in }}$ and the right antenna, the RTD oscillator power is reflected toward the left antenna. The left antenna receives the reflected power and radiates it outward. With two graphene antennas, the device radiates high harmonics to provide high-frequency $\mathrm{THz}$ waves.

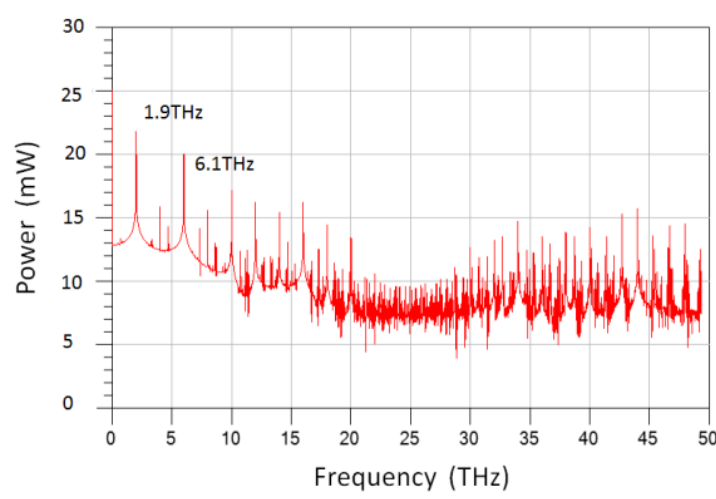

(a)

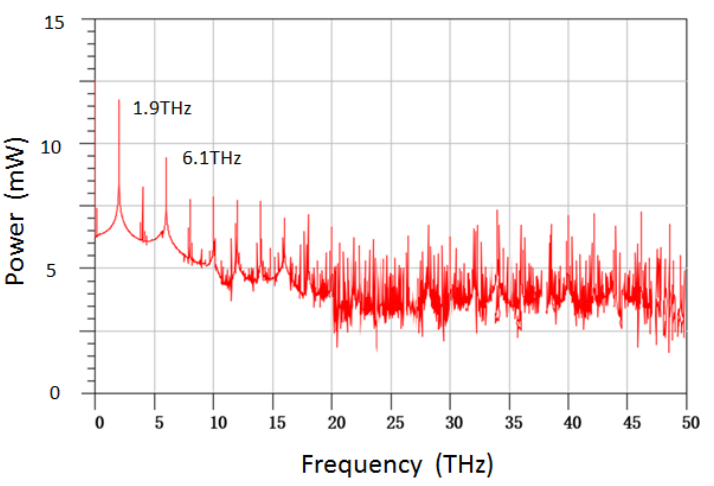

(b)

Figure 5. Power spectrum of oscillator: (a) Device dimensions are $L=2 \mu \mathrm{m}$ and $W=10 \mu \mathrm{m}$. (b) Device dimensions are $L=1 \mu \mathrm{m}$ and $W=10 \mu \mathrm{m}$.

\section{Conclusions}

The study herewith, described the design of a RTD oscillator with a RTD-gated-graphene-2DEF and demonstrated the functioning of this RTD oscillator through a fully distributed transmission line simulation model. This simulated work has developed the two main parts of the RTD Oscillator, the RTD and antenna. Amplification arising from the dynamics of electron plasma waves in a high electron mobility transistor (HEMT) channel with injection from the gate exhibiting negative differential resistance (NDR). In this paper, HEMT is replaced by a graphene layer, which has been proven to produce electron plasma. This study has proven that the impedance of the device changes periodically when the RTD electrical size $1 / 30 \leq k \leq 1 / 3$. As long as the impedance matching is achieved, the oscillation frequency is not limited by the size of the device. Therefore, without reducing the frequency, the output power can be greatly increased with the increase of the device size. At the same time, the graphene antenna provides two ultra-wide bands which are $1.37-1.93 \mathrm{THz}$ and $2.75-7.00 \mathrm{THz}$. Because of the mismatch between $Z^{\prime}{ }_{i n}$ and the right antenna, RTD oscillator power is reflected toward and radiated outward from the left antenna. Therefore, the proposed RTD oscillator can generate significantly higher levels of power than traditional RTD oscillators. With two graphene antennas, the device can radio high harmonics to provide RTD oscillator output powers of $22 \mathrm{~mW}$ at $1.9 \mathrm{THz}$ and $20 \mathrm{~mW}$ at $6.1 \mathrm{THz}$. The results of this research study can serve as a fundamental principle in the fabrication of a THz RTD oscillator. 
Author Contributions: Methodology, investigation, resources, and data curation, F.Z. and C.Z.; conceptualization, supervision, project administration, and funding acquisition, W.G.; validation, J.C.; writing-original draft preparation, writing - review and editing and visualization, C.A.T.H.T, L.S. and Y.Z.

Funding: This work was supported by the National Natural Science Foundation of China (No. 61331003).

Conflicts of Interest: The authors declare no conflict of interest.

\section{References}

1. Choe, W.; Jeong, J. A Broadband THz On-Chip Transition Using a Dipole Antenna with Integrated Balun. Electronics 2018, 7, 236. [CrossRef]

2. Kanaya, H.; Sogabe, R.; Maekawa, T.; Suzuki, S.; Asada, M. Fundamental Oscillation up to $1.42 \mathrm{THz}$ in Resonant Tunneling Diodes by Optimized Collector Spacer Thickness. J. Infrared Millim. Terahertz Waves 2014, 35, 425-431. [CrossRef]

3. Izumi, R.; Suzuki, S.; Asada, M. $1.98 \mathrm{THz}$ resonant-tunneling-diode oscillator with reduced conduction loss by thick antenna electrode. In Proceedings of the IEEE 2017 42nd International Conference on Infrared, Millimeter, and Terahertz Waves (IRMMW-THz), Cancun, Mexico, 27 August-1 September 2017; pp. 1-2. [CrossRef]

4. Orihashi, N.; Hattori, S.; Suzuki, S.; Asada, M. Experimental and Theoretical Characteristics of Sub-Terahertz and Terahertz Oscillations of Resonant Tunneling Diodes Integrated with Slot Antennas. Jpn. J. Appl. Phys. 2005, 44, 7809-7815. [CrossRef]

5. Li, C.H.; Chiu, T.Y. 340-GHz Low-Cost and High-Gain On-Chip Higher Order Mode Dielectric Resonator Antenna for THz Applications. IEEE Trans. Terahertz Sci. Technol. 2017, 7, 284-294. [CrossRef]

6. TeraView, TeraPulse Lx. Available online: http://www.teraview.com/products/TeraPulse\%204000/index.html (accessed on 13 September 2019).

7. TOPTICA Photonics, Terahertz Systems Optoelectronic Terahertz-Generation. Available online: https: //www.toptica.com/products/terahertz-systems/ (accessed on 13 September 2019).

8. Wasige, E.; Alharbi, K.H.; Alkhalidi, A.; Wang, J.; Khalid, A.; Rodrigues, G.C.; Figueiredo, J. Resonant tunnelling diode terahertz sources for broadband wireless communications. In Terahertz, RF, Millimeter, and Submillimeter-Wave Technology and Applications X; Society of Photo-optical Instrumentation Engineers: Bellingham, WA, USA, 2017; p. 101031J. [CrossRef]

9. Oshima, N.; Hashimoto, K.; Horikawa, D.; Suzuki, S.; Asada, M. Wireless data transmission of 30 Gbps at a 500-GHz range using resonant-tunneling-diode terahertz oscillator. In Proceedings of the 2016 IEEE MTT-S International Microwave Symposium (IMS), San Francisco, CA, USA, 22-27 May 2016; pp. 1-4. [CrossRef]

10. Maekawa, T.; Kanaya, H.; Suzuki, S.; Asada, M. Frequency increase in terahertz oscillation of resonant tunnelling diode up to $1.55 \mathrm{THz}$ by reduced slot-antenna length. Electron. Lett. 2014, 50, 1214-1216. [CrossRef]

11. Asada, M.; Suzuki, S.; Kishimoto, N. Resonant tunneling diodes for sub-terahertz and terahertz oscillators. Jpn. J. Appl. Phys. 2008, 47, 4375. [CrossRef]

12. Pozar, D.M. Microwave Engineering, 4 ed.; Wiley: Hoboken, NJ, USA, 2012; pp. 1-48.

13. Burke, P.J.; Spielman, I.B.; Eisenstein, J.P.; Pfeiffer, L.N.; West, K.W. High frequency conductivity of the high-mobility two-dimensional electron gas. Appl. Phys. Lett. 2000, 76, 745-747. [CrossRef]

14. Sensale-Rodriguez, B.; Lei, L.; Fay, P.; Jena, D.; Xing, H.G. Power amplification at THz via plasma wave excitation in RTD-gated HEMTs. IEEE Trans. Terahertz Sci. Technol. 2013, 3, 200-206. [CrossRef]

15. Sensale-Rodriguez, B. Graphene-insulator-graphene active plasmonic terahertz devices. Appl. Phys. Lett. 2013, 103, 123109. [CrossRef]

16. Mao, X.; Xie, S.; Zhu, C.; Geng, Z.; Chen, H. Theoretical study of terahertz active transmission line oscillator based on RTD-gated HEMT. AIP Adv. 2018, 8, 065323. [CrossRef]

17. Zhu, C.; Mao, L.; Zhao, F.; Mao, X.; Guo, W. Photo-Controlled Terahertz Amplified Modulator via Plasma Wave Excitation in ORTD-gated HEMTs. IEEE Photonics J. 2017, 9, 1-8. [CrossRef]

18. Chen, J.; Badioli, M.; Alonso-González, P.; Thongrattanasiri, S.; Huth, F.; Osmond, J.; Spasenović, M.; Centeno, A.; Pesquera, A.; Godignon, P. Optical nano-imaging of gate-tunable graphene plasmons. Nature 2012, 487, 77. [CrossRef] [PubMed]

19. Fei, Z.; Rodin, A.S.; Andreev, G.O.; Bao, W. Gate-tuning of graphene plasmons revealed by infrared nano-imaging. Nature 2012, 487, 82. [CrossRef] [PubMed] 
20. Thongrattanasiri, S.; Manjavacas, A.; de Abajo, F.J.G. Quantum Finite-Size Effects in Graphene Plasmons. ACS Nano 2012, 6, 1766-1775. [CrossRef]

21. Cox, J.D.; Silveiro, I.; de Abajo, F.J.G. Quantum Effects in the Nonlinear Response of Graphene Plasmons. ACS Nano 2016, 10, 1995-2003. [CrossRef]

22. Dasgupta, S.; Nidhi, Brown, D.F.; Wu, F.; Keller, S.; Speck, J.S.; Mishra, U.K. Ultralow nonalloyed Ohmic contact resistance to self aligned N-polar GaN high electron mobility transistors by $\operatorname{In}(\mathrm{Ga}) \mathrm{N}$ regrowth. Appl. Phys. Lett. 2010, 96, 3. [CrossRef]

23. Green, A.J.; Gillespie, J.K.; Fitch, R.C.; Walker, D.E.; Lindquist, M.; Crespo, A.; Brooks, D.; Beam, E.; Xie, A.; Kumar, V.; et al. ScAlN/GaN High-Electron-Mobility Transistors With 2.4-A/mm Current Density and 0.67-S/mm Transconductance. IEEE Electron Device Lett. 2019, 40, 1056-1059. [CrossRef]

24. Wang, R.H.; Saunier, P.; Xing, X.; Lian, C.X.; Gao, X.A.; Guo, S.P.; Snider, G.; Fay, P.; Jena, D.; Xing, H.L. Gate-Recessed Enhancement-Mode InAlN/AlN/GaN HEMTs With 1.9-A/mm Drain Current Density and 800-mS/mm Transconductance. IEEE Electron Device Lett. 2010, 31, 1383-1385. [CrossRef]

25. Guo, J.; Li, G.; Faria, F.; Cao, Y.; Wang, R.; Verma, J.; Gao, X.; Guo, S.; Beam, E.; Ketterson, A.; et al. MBE-Regrown Ohmics in InAIN HEMTs With a Regrowth Interface Resistance of 0.05 Omega mm. IEEE Electron Device Lett. 2012, 33, 525-527. [CrossRef]

26. Wang, J.; Al-Khalidi, A.; Zhang, W.; Wasige, E.; Kelly, A.E.; Watson, S.; Cantu, H.; Figueiredo, J. Resonant tunneling diode oscillators for optical communications. In Third International Conference on Applications of Optics and Photonics; International Society for Optics and Photonics: Bellingham, WA, USA, 2017. [CrossRef]

27. Cong, J.; Mao, L.; Xie, S.; Zhao, F.; Yan, D.; Guo, W. Photoelectric Dual Control Negative Differential Resistance Device Fabricated by Standard CMOS Process. IEEE Photonics J. 2019, 11. [CrossRef]

28. Yang, C.C. Influence of Parasitic Effects in Negative Differential Resistance Characteristics of Resonant Tunneling. Electronics 2019, 8, 673. [CrossRef]

29. Shanawani, M.; Masotti, D.; Costanzo, A. THz Rectennas and Their Design Rules. Electronics 2017, 6, 99. [CrossRef]

30. Suzuki, S.; Shiraishi, M.; Shibayama, H.; Asada, M. High-Power Operation of Terahertz Oscillators with Resonant Tunneling Diodes Using Impedance-Matched Antennas and Array Configuration. IEEE J. Sel. Top. Quantum Electron. 2013, 19, 8500108. [CrossRef]

31. Suzuki, S.; Teranishi, A.; Hinata, K.; Asada, M. Fundamental oscillation up to $831 \mathrm{GHz}$ in GaInAs/AlAs resonant tunneling diode. In Proceedings of the 2009 IEEE International Conference on Indium Phosphide \& Related Materials, Newport Beach, CA, USA, 10-14 May 2009. [CrossRef] 